\title{
Asuntos bioéticos relacionados con la planeación y respuesta ante la pandemia de COVID-19
}

\section{Ethical issues related to planning and responding to COVID-19 pandemic}

\author{
Ricardo F González-Fisher, ${ }^{*}$ Steve Rissman, ${ }^{*}$ Xavier González-Fisher ${ }^{\ddagger}$
}

Citar como: González-Fisher RF, Rissman S, González-Fisher. Asuntos bioéticos relacionados con la planeación y respuesta ante la pandemia de COVID-19. An Med (Mex). 2020; 65 (4): 288-300. https://dx.doi.org/10.35366/97467

\begin{abstract}
RESUMEN
La pandemia del novel coronavirus (COVID-19) es la amenaza de salud pública más grande del presente siglo, y se ha convertido en un reto para los sistemas de salud a nivel global. La planeación y respuesta a la pandemia generan cuestionamientos éticos importantes en relación con el liderazgo, la limitación de libertades individuales, la necesidad de racionar los cuidados de salud en el contexto de una escasez de recursos, el uso de terapias experimentales y el riesgo que enfrentan los trabajadores de la salud.
\end{abstract}

Palabras clave: COVID-19, triaje, terapia experimental, ética, bioética.

Nivel de evidencia: III

\begin{abstract}
The novel coronavirus (COVID-19) is the world's largest public health threat, that is turning into a challenge for health systems worldwide. Planning and responding to this pandemic originate important ethical considerations related to leadership, limitation to personal freedom, the need to ration healthcare in a context of scarce resources, the use of experimental therapy and the risks that healthcare workers face.
\end{abstract}

Keywords: COVID-19, triage, experimental therapy, ethics, bioethics.

Level of evidence: III

\section{INTRODUCCIÓN}

En el siglo XX surgieron tres pandemias notables de influenza: la "gripe española (virus H1N1) en 1918 y 1919, con aproximadamente 50 millones de muertes a nivel global; la «influenza asiática» (virus H2N2) en 1957-1958, que dio como resultado entre uno y dos millones de muertes en el mundo; y la «influenza de Hong Kong» (virus H3N2) que produjo un millón de muertes en $1968 .{ }^{1}$ Debido a la globalización y al aumento en los viajes internacionales, los expertos en salud pública habían anticipado que el siguiente azote mundial aparecería en el siglo XXI. En el año 2003, un brote del síndrome respiratorio agudo grave (SARS, por sus siglas en inglés) infectó a más de

\footnotetext{
* Department of Health professions, Metropolitan State University of Denver. Estados Unidos.

₹ Notario Público. Profesor de Derecho Civil en la Universidad Panamericana campus Aguascalientes. Miembro del claustro académico del Colegio Nacional del Notariado Mexicano. México.

Recibido para publicación: 25/05/2020. Aceptado: 21/07/2020. 
8,000 personas a lo largo de cinco meses, el cual fue causado por un nuevo coronavirus humano surgido en áreas rurales de China y se esparció a cinco países en cuestión de días, diseminándose a 30 países en unos cuantos meses. Fallecieron $10 \%$ de las personas expuestas al virus. El SARS demostró la velocidad con la que un virus o cualquier agente infeccioso similar puede viajar a través del mundo. El resurgimiento del virus H1N1 durante la temporada de influenza 2009-2010, la aparición del Coronavirus del Síndrome Respiratorio del Oriente Medio (MERSCoV, por sus siglas en inglés) en 2012, y los casos de enfermedades severas en humanos causadas por la nueva influenza aviar acentuaron las preocupaciones existentes por la necesidad de estar preparados para las pandemias. ${ }^{2}$

Una pandemia viral sucede cuando surge un nuevo virus que se disemina fácilmente a nivel global. Ante la ausencia de memoria inmunológica en la población y sin una vacuna disponible, las personas pueden enfermar de gravedad. ${ }^{3}$ En el peor de los escenarios, una pandemia similar a la ocurrida en 1918 atacaría simultáneamente en extensas regiones del mundo con infecciones activas de varias semanas de duración, obligando a las comunidades a depender de sus propias reservas; ${ }^{4}$ el desarrollo de vacunas podría ocurrir sólo después de que una gran parte de la población se haya expuesto al virus, por lo que se podrá hacer muy poco para mitigar la morbilidad y mortalidad iniciales. Una pandemia de esta naturaleza puede durar entre 12 y 36 meses, atacando en oleadas de seis a ocho semanas, separadas entre sí por periodos de varios meses, durante los cuales el virus podría mutar y ser más fácilmente transmisible a los humanos. La aparición de un brote durante la temporada de influenza estacional generaría confusión entre la población con la consecuente sobreutilización de recursos de salud. ${ }^{3}$

Existen dos estrategias fundamentales para controlar una pandemia. Las políticas de mitigación, enfocadas a hacer que la propagación de la enfermedad sea más lenta, reduciendo, en consecuencia, picos en la demanda de servicios de salud mientras se protege a los más vulnerables, lo que se conoce como «aplanar la curva»; en tanto que las políticas de supresión buscan controlar el brote epidémico inicial mediante la reducción del número de casos manteniendo la situación por periodo indefinido..$^{5,6}$

La pandemia del novel coronavirus (COVID-19) es la amenaza de salud pública más grande en lo que va del presente siglo. Se ha esparcido por casi todos los países; en el periodo comprendido entre el 31 de diciembre de 2019 y el 26 de septiembre de 2020 se habían reportado 32,613,844 casos de COVID-19 (de acuerdo con las definiciones de casos y estrategias de aplicación de pruebas en los países afectados) y 989,728 muertos por la enfermedad. ${ }^{7}$ No se había estudiado a muchas personas más, por lo que es difícil determinar exactamente el número de personas infectadas. ${ }^{8}$ De igual forma, se ha sugerido que puede haber un subregistro en el número de fallecimientos, ya que el número de muertes no atribuidas a COVID-19 ha sido mucho mayor que el número de muertes esperado en 32 países. ${ }^{9}$ Por lo anterior, en un escenario similar a la pandemia de 1918, se estima que entre 15 y $35 \%$ de la población mundial podría resultar infectada con COVID-19 y, la mortalidad podría alcanzar los 100 millones de personas a nivel global. Algunos cálculos estiman que podría haber 90 millones de personas afectadas y dos millones de muertes tan sólo en los Estados Unidos. El número de pacientes hospitalizados en ese país fluctuará entre 865,000 y 9.9 millones. ${ }^{10}$

Esta pandemia representa un reto para los sistemas de servicios de salud a nivel global, y genera cuestionamientos éticos importantes, en especial en relación con la posible necesidad de racionar los cuidados de salud en el contexto de una escasez de recursos y capacidad instalada para resolver la crisis. Aun si la capacidad de brindar cuidados fuera suficiente, será prioritario establecer metas ante el escenario de enfermedades agudas que pongan en peligro la vida, especialmente en aquellos pacientes con enfermedades crónicas, mientras que se intente, en la medida de lo posible, alinear la atención disponible con los valores y expectativas de los pacientes.

\section{PREPARACIÓN ÉTICA}

Hasta antes de la mitad de la década de los 70 del siglo pasado, el medicus dixit era la norma a seguir en el tratamiento de los pacientes. La verticalidad y el paternalismo en la toma de decisiones era lo que primaba y el juicio facultativo difícilmente era cuestionado. A partir de ese momento surgieron voces demandando primero, tomar en cuenta el parecer de la persona que recibe el servicio sanitario y posteriormente, el espectro de toma de decisiones se ampliaría también hacia quienes se asumen como "responsables del enfermo" o simplemente forman parte de su entorno. Y es que la sociedad ha estado expuesta a una mejor educación y a una gran cantidad de información, esta última quizás no de calidad, pero información al fin. ${ }^{11}$ En 1979 Childress y Beauchamp 
establecieron cuatro principios éticos fundamentales obligatorios (prima facie) que definen actualmente el quehacer de los proveedores de servicios de salud, éstos son: 1) respeto a la autonomía del paciente: de donde deriva, entre otras cosas, el consentimiento informado; 2) beneficencia: que las acciones médicas sean en beneficio del paciente; 3) no maleficencia: basada en el principio hipocrático primum non nocere; y 4) justicia: la distribución equitativa de los recursos finitos de salud. ${ }^{12} \mathrm{El}$ apego a estos principios es de suma importancia cuando se enfrenta una emergencia sanitaria de gran magnitud.

Los efectos del SARS a principios de 2003 destacaron la importancia de establecer con anticipación un marco de trabajo ético para la toma de decisiones en caso de cualquier desastre médico predecible. Durante esta crisis no se describieron de manera explícita las presuposiciones éticas al planear e implementar acciones de salud pública, por lo que surgieron varios problemas sociales como la pérdida de la confianza de la población, baja motivación del personal hospitalario, confusión en los roles y responsabilidades, estigmatización de comunidades vulnerables así como diseminación de información incorrecta. ${ }^{2}$

Para evitar problemas similares, a partir de 2006, varias organizaciones internacionales se han enfocado en las consideraciones éticas relacionadas con la planeación para las pandemias.

En febrero de 2007 el Centro para el Control y Prevención de las Enfermedades en los Estados Unidos (CDC, por sus siglas en inglés) estableció una serie de guías y principios para la toma de decisiones públicas para enfrentar una futura pandemia de influenza. ${ }^{2}$ El documento define varios puntos de acción que pueden servir como base para enfrentar éticamente cualquier crisis de salud pública global, éstos incluyen:

- Un compromiso general a la transparencia en el proceso de toma de decisiones.

- La participación de grupos diversos y transversales del público en dicha toma de decisiones, con atención especial a las comunidades históricamente marginadas.

- Maximizar la preparación.

- Usar la mejor evidencia científica disponible.

- Establecimiento de redes globales que se esfuercen en buscar el bienestar común.

- Un balance entre la libertad individual y los intereses comunes, incluyendo las restricciones a las libertades individuales para proteger a los más afectados.
- Justicia en los procesos de decisión (justicia procesal).

Para responder a la interrogante sobre los riesgos que debe asumir el personal de salud y su responsabilidad con su propio bienestar y el de sus familias, en 2010, la Administración de Veteranos (VA, por sus siglas en inglés) ${ }^{13}$ afirmó que sus empleados «tienen la obligación de brindar cuidados a los pacientes, aun cuando esto implique cierto riesgo personal». Sin embargo, la agencia también definió las acciones que deben ser tomadas en sus instalaciones para minimizar ese riesgo, y permitir que los empleados cumplan con sus obligaciones. Éstas incluyen:

- Asegurar que las necesidades humanas básicas (por ejemplo, alimentos, agua, descanso) puedan ser satisfechas durante las horas de trabajo.

- Proporcionar vacunas, medicamentos antivirales, equipo de protección personal y otras medidas que disminuyan los riesgos laborales.

- Brindar los medios suficientes para garantizar la seguridad personal.

- Apoyar al personal a cumplir con sus responsabilidades personales, incluyendo el cuidado de niños o personas dependientes, en la medida de lo posible.

- Asegurar el acceso a recursos médicos para el personal enfermo, en la medida de lo posible.

De ambos documentos se desprende que ante la amenaza potencial y la dificultad de predecir cuándo ocurrirá la siguiente pandemia, la planeación se convierte en una obligación.

\section{LA OBLIGACIÓN DE PLANEAR}

A diferencia de lo que sucede en situaciones clínicas habituales, la medicina por sí sola puede resultar insuficiente para tomar decisiones de salud durante una pandemia. En una crisis de tal magnitud se presentan dos prioridades éticas principales: la prioridad de salvar vidas y la prioridad de mantener el funcionamiento de la sociedad. Ante estas circunstancias los profesionales de la salud enfrentarán conflictos morales al no poder brindar servicios de la manera habitual, puesto que los recursos estarán sumamente comprometidos, por lo que en salud pública la discusión ética se enfoca en resolver conflictos sobre acciones que afectan a la sociedad cuando se toman medidas para prevenir enfermedades, prolongar la vida, o promover el bienestar biopsicosocial en la comunidad. ${ }^{14}$ 
De acuerdo con la CDC, la centralización en la toma de decisiones permite facilitar el seguimiento de la enfermedad, conservar el funcionamiento social en todas las comunidades así como asignar recursos limitados (por ejemplo, vacunas, medicamentos antivirales, y el tiempo de los profesionales de salud) con un énfasis en el principio ético de justicia. Esta centralización debe considerar las consecuencias de las medidas implementadas con principal atención al respeto a las personas, la no maleficencia y la justicia.

La centralización en el control de la pandemia debe garantizar que los recursos alcancen también a las áreas más vulnerables como las de menores ingresos, vecindarios sobrepoblados en grandes ciudades o áreas rurales dispersas. Para estos casos se considera adecuado crear centros de transición que brinden atención a personas que no cuenten con recursos suficientes para convalecer en casa, estos centros deberán contar con personal preparado y camas equipadas con el fin de aliviar la presión de los grandes hospitales. ${ }^{4,15}$

Debido a las condiciones generadas por la pandemia de COVID-19 los servicios de salud se encuentran en lo que se conoce como «entorno VUCA», situación que se caracteriza por la volatilidad, la incertidumbre, la complejidad y la ambigüedad. Este concepto fue fraguado en la década de los 90 por el ejército estadounidense y responde al acrónimo inglés formado por los términos Volatility (V), Uncertainty (U), Complexity (C) y Ambiguity (A). ${ }^{16}$

Estos escenarios nos obligan a adaptarnos a cambios continuos en programación estratégica y rutinas profesionales. A diferencia de lo que sucede en un entorno clínico habitual, en circunstancias de crisis los miembros del equipo de salud requerirán indicaciones precisas y claras, ante lo cual, el líder deberá aplicar su experiencia en el manejo de la ambigüedad y la incertidumbre.

Algunas consideraciones que debe tener el líder en momentos de crisis:

a) Poner atención a la parte inferior de la pirámide de Maslow: una vez satisfechas las necesidades primarias de los miembros del equipo, podrán alinearse para un objetivo común e incluso crecer durante la crisis.

b) Anticipar las consecuencias no intencionales de la crisis como el impacto económico, social y financiero para nuestro equipo y nuestros pacientes.

c) Recordar que en momentos de crisis lo importante se vuelve urgente y lo urgente crítico, por lo que el líder deberá delegar lo urgente empoderando a otros a ser líderes bajo un propósito común. d) Simultáneamente el líder deberá conectar, motivar e inspirar siendo empático. En casos de guerra los mandos militares anteponen el bienestar y la seguridad de otros al suyo propio, es decir, anteponen la humildad y responsabilidad sobre la arrogancia y bravuconería.

e) El líder deberá saber proponer acciones precisas ante situaciones inciertas. Los problemas complejos y las crisis son magníficas oportunidades para aprender. Los líderes deben mantener un estado de alerta constante así como interpretar y sintetizar experiencias previas que puedan aplicarse en tiempo real. Al comparar lo «desconocido» de la crisis actual con lo «vivido» en crisis anteriores el líder podrá poner las cosas en perspectiva, identificar patrones y unir los puntos para determinar un plan de acción. ${ }^{17}$

La ética tiene un papel fundamental en la comunicación en tiempos de crisis. En crisis epidemiológicas el líder deberá brindar la información y orientación necesarias para que el público y otros actores puedan responder de manera adecuada. ${ }^{18}$ La comunicación, incluyendo interacciones verbales, visuales y escritas entre la organización y sus miembros antes, durante y después de un impacto negativo deberá enfocarse en un evento específico, identificar consecuencias probables y brindar información para controlar daños en comunidades afectadas. La comunicación debe ser honesta, oportuna, sencilla, certera y completa. ${ }^{19}$

En un estudio reciente sobre comunicación masiva sobre COVID-19 en medios electrónicos se encontró que $27.5 \%$ de los videos publicados en YouTube contenían información falsa originada en noticias de entretenimiento, noticias de internet y otros usuarios, alcanzando a 62 millones de personas a nivel mundial. ${ }^{11}$

Sabiendo que la información puede generar efectos negativos, sobre todo si se conduce de manera inadecuada, es indispensable someterla a un escrutinio riguroso ${ }^{18}$ reevaluando constantemente para tener la certeza de que está alineada con el plan de acción. Para esto, un líder debe ser su mayor crítico. ${ }^{20} \mathrm{De}-$ bido a que la relación médico-paciente es asimétrica con base en conocimientos, es importante que los médicos evalúen cuidadosamente la información que publican en sus redes sociales. ${ }^{14}$

\section{MEDIDAS DE RESTRICCIÓN (AUTONOMÍA Y EL BIEN COMÚN)}

Durante una pandemia puede ser necesario aplicar ciertas medidas restrictivas para promover la salud 
pública, estas restricciones incluyen el aislamiento de personas infectadas, la cuarentena de los expuestos, cierres escolares y de eventos públicos (por ejemplo, en estadios deportivos), restringir el acceso a lugares públicos (por ejemplo, supermercados o tiendas de alimentos), flexibilizar opciones de trabajo y limitaciones para viajar.

Las medidas restrictivas son éticamente justificables sólo en el caso de que no aplicarlas afecte de manera importante el funcionamiento de la sociedad o el bienestar público. ${ }^{21} \mathrm{Su}$ aplicación debe considerarse al principio de la pandemia, procurando, de ser posible, sean asumidas de manera voluntaria. La aplicación obligatoria debe ser justificada a través de un proceso público transparente con base en la mejor evidencia científica disponible. En este caso deben ser aplicadas de manera equitativa, evitando invasiones innecesarias a la privacidad y acciones que inciten la estigmatización.

La aplicación de medidas restrictivas por periodos largos puede generar falta de ingresos y otras carencias, afectando desproporcionalmente a los más pobres, por lo que CDC recomienda que, ante la aplicación de medidas restrictivas, se implementen acciones que garanticen el acceso a servicios esenciales (alimentos, agua, seguridad laboral y apoyo) para cumplir con obligaciones económicas.

Por último, se debe planear para cuando llegue el momento de clausurar la infraestructura rediseñada para tratar los casos de COVID-19, ya que la atención de pacientes cuyos tratamientos electivos tengan que posponerse, causará una nueva oleada de estrés al sistema. Un liderazgo proactivo será clave para evitar conflictos internos al priorizar recursos, y volver de manera sutil a la normalidad. ${ }^{22}$

\section{DISTRIBUCIÓN DE RECURSOS LIMITADOS}

Las enfermedades infecciosas como el SARS y la influenza pandémica han alertado a los servicios de emergencia sobre la necesidad de que sus planes de contingencia consideren que para maximizar los resultados utilizando los recursos disponibles en emergencias de salud pública, los sistemas de salud deben enfocarse en el cuidado de la población más que en el cuidado del individuo. ${ }^{23}$ Esto es particularmente difícil durante una pandemia cuando la capacidad de los hospitales llegue a su límite y no pueda extenderse para cubrir la demanda.

Cuando las necesidades existentes no pueden ser cubiertas con los recursos disponibles, los servicios de salud utilizan el triaje, un sistema cuyo objetivo es mejorar la calidad de la atención priorizando casos de acuerdo con lineamientos predeterminados.

\section{a) Lineamientos del triaje}

Triage es un término derivado de la palabra francesa trier (clasificar). Originalmente se utilizó para la clasificación de productos agrícolas, en la actualidad se usa de manera casi exclusiva en contextos específicos del cuidado de la salud como al decidir a quién admitir a salas de emergencia o unidades de cuidados intensivos; listas de espera para tratamientos que salvan la vida como los trasplantes de órganos y en situaciones en el campo de batalla. La distribución de servicios médicos y hospitalarios durante las pandemias debe también incluirse en las discusiones generales de triaje..$^{4,23}$

Las decisiones que limitan el acceso a los tratamientos fundamentales e incluso aquéllas de vida o muerte están rodeadas de controversia y resultan difíciles de tomar para los profesionales de la salud y aún más difíciles de entender por parte de los pacientes.

Con frecuencia los profesionales de la salud no cuentan con entrenamiento suficiente para analizar bajo presión y con tiempo limitado los múltiples factores involucrados en las decisiones de triaje. Por su parte, los pacientes, justificadamente, tienen una visión egocéntrica y limitada en lo referente a la atención de su salud, en consecuencia, tanto el clínico como el paciente pueden considerar que el triaje es inmoral. Educar sobre triaje de manera proactiva y evaluar su aplicación en el contexto de la situación ayudará a los involucrados a comprender mejor las consideraciones, consecuencias y principios éticos de dicho proceso. ${ }^{24}$

El proceso de triaje se basa en tres elementos: 1) que exista escasez, por lo menos moderada, de recursos; 2) que un profesional del cuidado de la salud (con frecuencia denominado «oficial del triaje») evalúe las necesidades médicas de cada paciente a través de un examen breve; y 3) que exista un sistema o plan de acción basado en criterios y algoritmos que guíen al «oficial de triaje» en determinar la prioridad de tratamiento para cada paciente.

La aplicación del triaje requiere de altos niveles de práctica, destreza y madurez profesional. ${ }^{24,25}$

Desde el punto de vista de las teorías de la ética, el triaje es un ejemplo clásico de justicia distributiva que se enfoca en cómo distribuir los beneficios y las cargas entre la población. Pero su discusión debe incluir además los principios éticos de no maleficencia, beneficencia, fidelidad, veracidad y autonomía 
y justicia, ${ }^{24}$ además de evaluar el conflicto ético entre los principios utilitarios de hacer el mayor bien para el mayor número de personas, el principio de igualdad de respeto para todos y el principio de la no maleficencia contra el principio del no abandono del paciente. ${ }^{21}$

El principio ético de la no maleficencia se desprende del Juramento Hipocrático que menciona: Estableceré el régimen de los enfermos de la manera que les sea más provechosa según mis facultades y a mi entender, evitando todo mal y toda injusticia. ${ }^{26}$ Durante situaciones de desastre, el objetivo de las acciones de salud es asegurar recursos y medidas de protección equitativas y justas para los grupos vulnerables, al mismo tiempo los profesionales de la salud tienen la obligación de brindar los mejores cuidados posibles de acuerdo con las circunstancias. Sin embargo, periodos de espera prolongados pueden incrementar el dolor y el sufrimiento así como afectar negativamente los resultados de tratamientos ocasionando daños indirectos incluyendo riesgo de daño psicosocial -estrés, miedo, sensación de descuido o de abandono-. ${ }^{27}$

La falta de comunicación en cuanto al triaje se ha asociado con actitudes agresivas y violentas de pacientes y acompañantes contra personal de urgencias ${ }^{28}$ en consecuencia, Ekwall y colaboradores ${ }^{29}$ han subrayado la importancia de incluir las necesidades psicosociales de los pacientes a lo largo del proceso del triaje. No hacerlo puede comprometer el principio de no maleficencia. ${ }^{23}$

El principio ético de beneficencia -que las acciones médicas se ejerzan con el único fin de beneficiar al paciente- debe tomarse en cuenta en el diseño del triaje. Ante una situación que involucre la distribución de recursos limitados de salud, puede surgir la necesidad de afectar el bien de un paciente para asegurar el de otros. Esta tarea necesaria e incómoda corresponde al médico. En el escenario de una pandemia, la obligación de «hacer el mayor bien a la mayor cantidad de personas» depende de diferentes variables, por lo que es imposible establecer reglas y lineamientos que aseguren la aplicación del principio de beneficencia para todas las personas en una situación de triaje. La comparación de los resultados individuales de cada paciente con la predicción de los resultados esperados demostrará la beneficencia para cada paciente y para el grupo en su totalidad. ${ }^{24}$

Ya que la confianza en la relación profesional se basa en el principio ético de fidelidad, durante episodios de triaje el médico puede experimentar conflictos entre acciones que sirven al bien social y la atención de un paciente individual. La comunicación con respecto al triaje puede reforzar la confianza, sobre todo si el paciente comprende que el retraso en su atención se debe a que su médico está atendiendo a otros pacientes con necesidades más urgentes, y al grupo en su totalidad, de esta forma paciente y médico podrán participar juntos de manera altruista para lograr el beneficio de otros pacientes y del grupo. ${ }^{24}$

Por otra parte, la comunicación además es importante para reforzar el principio ético de veracidad que consiste en cumplir con la expectativa que tienen los pacientes de que se les comunique la verdad. Sin veracidad no puede haber fidelidad, y en consecuencia no podrá haber confianza, por lo que las «cláusulas de restricción de información» (gag clauses) relacionadas con los médicos y los lineamientos del triaje durante una pandemia no son éticamente aceptables.

El respeto a la autonomía es un criterio fundamental para la toma de decisiones en servicios de salud. Se refiere a que las personas competentes tienen el derecho de tomar decisiones relacionadas con su propia atención médica. ${ }^{23,24}$ Respetar la autonomía del paciente incluye su derecho a la privacidad, respeto a sus decisiones, autodominio, su derecho a escoger libremente entre diferentes opciones de tratamiento, respeto a las convicciones y postura moral del paciente y que el paciente acepte la responsabilidad por sus propias decisiones. ${ }^{12}$

Durante situaciones extremas, algunos pacientes reconocen de manera inmediata la necesidad de llevar a cabo el triaje y eligen cooperar de manera voluntaria, incluso ayudando con los cuidados de otros pacientes, considerados de mayor urgencia. A través de la educación de la sociedad con respecto a las necesidades individuales, y las necesidades colectivas durante situaciones de triaje, será factible que más personas entren a esta categoría, y será más fácil respetar la autonomía individual. Desafortunadamente, el respeto por la autonomía individual no siempre puede aplicarse como en el caso de niños o pacientes severamente enfermos que carecen del autodominio y el razonamiento moral que los hace incapaces de decidir lo mejor para sí mismos desde el punto de vista médico. De manera similar, durante el triaje, la autonomía de algunos individuos puede ser limitada si no comprenden la diferencia entre el bien personal $\mathrm{y}$ el bien colectivo. ${ }^{24}$

El principio ético de justicia, entendido de manera más específica como justicia distributiva, requiere que el reparto de ciertos recursos limitados se lleve a cabo de manera equitativa, al igual que la distribución de la carga y los beneficios. Los principios del 
triaje distribuyen los beneficios de recibir cuidados de salud, y la carga de retrasar o diferir la atención entre los miembros enfermos de una población. Esto no significa que cada persona o grupo deba recibir una parte igual de los recursos escasos (igualdad), sino más bien una parte justa, basada en criterios y principios apropiados (equidad) previamente establecidos. ${ }^{30}$ En una situación de restricciones en la distribución de servicios de salud que pueden afectar incluso la vida y la muerte, un triaje explícito es quizá la única forma en que los individuos puedan confiar en su médico y liberarse de las preocupaciones relacionadas con su autonomía.

Cuando no existen lineamientos claros, concisos y explícitos, el triaje puede ser visto como un proceso inadecuado y desorganizado. Las guías clínicas pueden ser útiles para promover buenas prácticas éticas durante estas situaciones, pero no deben sustituir el razonamiento médico. El oficial del triaje deberá tomar decisiones de alto impacto de manera rápida y con información limitada. Al ser ejecutado por una sola persona el proceso podrá ser sujeto a críticas con base en sesgos y desigualdades; la inclusión de un sistema de auditoría en los lineamientos de triaje promoverá el apego al principio de justicia en estas situaciones. ${ }^{24}$

\section{b) Ventiladores y soporte vital}

En circunstancias clínicas habituales, todo paciente que requiere de un ventilador a causa de condiciones potencialmente reversibles lo recibe, a menos que el paciente o sus subrogados, lo rechacen. Sin embargo, se ha visto en diversos países que esto no será posible durante una pandemia, y algunos pacientes que podrían sobrevivir con el apoyo de un ventilador, morirán si no hay alguno disponible. Ante esta emergencia de salud pública, los hospitales y los estados deberán establecer e implementar, de manera urgente, políticas que distribuyan los recursos escasos con apego al principio de justicia, y que conforten a las familias de los pacientes que fallecen. ${ }^{31}$

Las recomendaciones existentes para la distribución de recursos limitados de cuidados críticos incluyen condiciones éticamente problemáticas como la exclusión categórica de ciertos grupos de pacientes.

De acuerdo con lineamientos hospitalarios y recomendaciones de algunas organizaciones profesionales, algunos pacientes son excluidos del acceso a unidades de cuidados intensivos (UCI) debido a la preexistencia de condiciones comórbidas (falla cardiaca clase III o IV, enfermedades pulmonares crónicas, enfermedad renal terminal, discapacidades cognitivas severas, entre otras). Aun si estas exclusiones se aplicaran de forma explícita, se consideran éticamente sesgadas, ya que los criterios de exclusión (pronóstico a largo plazo y estado funcional) se aplican de manera selectiva sólo a cierto grupo de personas y no a todos los pacientes que pudieran recibir cuidados críticos, lo cual viola el principio ético de justicia. Este tipo de exclusiones tampoco se justifica durante una emergencia de salud pública.

Excluir categóricamente a un grupo de pacientes puede generar una percepción de discriminación al hacer sentir a muchos que no vale la pena salvar sus vidas. Más aún, las exclusiones categóricas son demasiado rígidas ante una crisis de naturaleza dinámica, en la que la carencia de ventiladores surgirá y disminuirá en oleadas durante la pandemia. ${ }^{32}$

Un enfoque frecuentemente recomendado para distribuir ventiladores en momentos de escasez consiste en dar prioridad a aquellos pacientes críticamente enfermos que tienen mayores posibilidades de sobrevivir al ser dados de alta del hospital con tratamiento. Aunque relevante, esta forma de tratar de hacer el mayor bien al mayor número de personas puede resultar inadecuado porque ignora otras consideraciones éticamente importantes como tomar en cuenta el número de años de vida salvados; este criterio se aplica para la distribución de pulmones para trasplantes en los Estados Unidos, que incorpora la duración esperada de la sobrevivencia después del trasplante, y no solamente la posibilidad de que el trasplante evite la muerte. ${ }^{31}$

Las personas que desempeñan actividades esenciales para salvar vidas durante la pandemia, como los profesionales de salud y los rescatistas, también deben tener prioridad en la asignación de recursos limitados. Esta prioridad no sólo se debe a su valor intrínseco, sino a su valor instrumental para salvar a otros. Dicha prioridad también puede considerarse una manera de reciprocidad al hecho de que estos servidores se ponen en riesgo para ayudar a otros. Debería entonces hacerse explícito que en caso de escasez los ventiladores no serán distribuidos con base en consideraciones moralmente irrelevantes como sexo, raza, religión, discapacidad intelectual, capacidad adquisitiva, ciudadanía, estrato socioeconómico o relaciones sociales. ${ }^{31}$

Ante la ausencia de un criterio único que abarque todos los valores moralmente relevantes, se deberán integrar varios criterios en una herramienta única para dar prioridad a cuáles pacientes deben recibir los ventiladores en momentos en los que no haya suficientes para todos. 
En marzo de 2020, con base en las opiniones de diversos grupos de ciudadanos, expertos en ética y medicina en Pennsylvania, EUA, se aprobó una política que a su vez ha sido seguida por otros estados de la Unión Americana. Esta política no excluye de manera categórica a grandes grupos de pacientes, pero permite dar prioridad a aquéllos que obtendrían un mayor beneficio y al mismo tiempo puede ser consistentemente aplicada durante cambios rápidos en la disponibilidad de ventiladores. ${ }^{33}$

Con base en la meta de maximizar los resultados en la población, las guías existentes reconocen la necesidad de «redistribuir» ventiladores cuando se excede la capacidad, pero generalmente estas guías no resuelven la incertidumbre médica ni las complejidades psicológicas de esta acción. ${ }^{31}$ La justificación ética para reubicar un ventilador es que el beneficio social se verá afectado ante el uso indefinido de ventiladores por pacientes con pocas posibilidades de sobrevivir.

Una manera de reducir el impacto emocional que la escasez de ventiladores puede causar en el personal de salud, los pacientes y sus familias es establecer expectativas adecuadas a priori, en las que el uso de los ventiladores mecánicos se presente como una medida terapéutica temporal y no ilimitada, esta «temporalidad» no debe ser demasiado breve, para evitar «ciclos rápidos» de pacientes que son retirados del ventilador y que, de haber sido tratados durante más tiempo, hubieran sobrevivido. ${ }^{34}$ Es recomendable nombrar a un oficial de triaje o un equipo que tome las decisiones sobre distribución, retiro y reubicación de estos equipos, esto aportará objetividad, evitará conflictos morales y minimizará el sufrimiento de los médicos a cargo. El uso de cuidados paliativos y medidas de confort deberá ser prioritario para aquellos pacientes que sean retirados del ventilador.

Ante la escasez de recursos, algunos centros hospitalarios han resaltado la importancia de implementar órdenes de no resucitación para ciertos pacientes hospitalizados durante la pandemia.

La discusión sobre el uso de maniobras avanzadas de reanimación cardiopulmonar en pacientes hospitalizados es importante para brindar tratamientos concordantes con los deseos de los pacientes. Esto adquiere mayor relevancia durante la pandemia por tres razones: primero, los médicos deben evitar medidas extremas para conservar la vida a pacientes que han expresado no aceptarlas. Segundo, evitar cuidados indeseados o con poco beneficio se vuelve especialmente importante en tiempos de estrés de la capacidad del sistema de salud. Tercero, brindar cuidados no aceptados o que no puedan ser de beneficio puede incrementar el riesgo de infección en otros pacientes, familiares y profesionales de la salud. ${ }^{32}$

Además, la aplicación de maniobras fútiles tendrá un impacto psicológico en los familiares del paciente y pondrá en riesgo los recursos disponibles de equipo de protección personal.

La implementación de órdenes de no resucitación se puede aplicar en diferentes situaciones, por ejemplo, por comunicación expresa del paciente o sus subrogados, esta decisión puede basarse en la recomendación del médico a través de un consentimiento informado. ${ }^{33}$

En situaciones extremas y bajo ciertas circunstancias en las que la resucitación avanzada no tenga posibilidad de ser efectiva, los médicos pueden indicar la «no resucitación» de forma unilateral. Este enfoque no es aceptado de manera universal, pero esta opción puede considerarse durante una pandemia. ${ }^{34}$

Brindar confort al final de la vida es difícil cuando los pacientes con COVID-19 están en aislamiento para prevenir la transmisión del virus. Debido a que los pacientes con frecuencia se manejan en aislamiento el contacto con sus seres queridos puede ser mínimo o inexistente, lo cual puede incrementar la ansiedad y otros problemas emocionales, asimismo la falta de oportunidad de despedirse puede generar un duelo complicado. ${ }^{35}$ De acuerdo con los lineamientos de la European Respiratory Society para cuidados paliativos de pacientes con COVID-19 publicados en julio de 2020, se debe permitir la visita de familiares o seres queridos a pacientes moribundos con COVID-19, y en caso necesario se les debe proporcionar equipo personal de protección. ${ }^{36}$ Las interacciones virtuales de la familia con el moribundo no ha sido evaluadas, e incluso algunos autores han sugerido que este tipo de contacto en tiempos de pandemia podría generar angustia, ${ }^{37}$ mientras que otros lo recomiendan. ${ }^{38}$ Los profesionales de la salud también podrán necesitar apoyo emocional. ${ }^{39}$

\section{CUIDADOS PARA EL EQUIPO DE PROFESIONALES DE LA SALUD}

Todo sistema de salud resultará severamente comprometido durante una pandemia, y la incapacidad de brindar los cuidados éticamente requeridos puede tener un impacto negativo en el bienestar moral y mental del personal de salud, por lo que se deberá recordar que el objetivo durante la emergencia sanitaria es salvar el mayor número de vidas posible manteniendo el funcionamiento de la sociedad.

Más allá de la justicia social y la distribución de recursos, surgen otras consideraciones éticas im- 
portantes a partir de la pandemia, incluyendo los retos de balancear los derechos individuales y las necesidades de salud de la comunidad frente a la obligación de los profesionales de la salud de brindar cuidados en diversos escenarios del brote de una enfermedad infecciosa. ${ }^{4}$

De acuerdo con datos de CDC, el 28 de febrero de 2016 había 28,639 casos y 11,316 muertes por la epidemia de ébola en el oeste de África en 2014. Los profesionales de la salud al cuidado de estos pacientes constituyeron uno de los grupos de mayor riesgo de contraer la enfermedad. Desde el inicio de la epidemia hasta noviembre de 2015 se confirmaron 881 infecciones y 513 defunciones en personal de salud de Guinea, Liberia y Sierra Leona. Liberia perdió 8\% de sus médicos, personal de enfermería y parteras, mientras que Sierra Leona y Guinea perdieron $7 \%$ y $1 \%$ de sus trabajadores de la salud respectivamente. ${ }^{40}$

La pandemia de COVID-19 puede tener efectos similares. De acuerdo con datos de la CDC, 50\% de los casos confirmados adquiridos por exposición directa con enfermos (222 de 445 ) hasta el 26 de febrero de 2020 en Estados Unidos eran personal de salud. ${ }^{41}$ En un estudio prospectivo observacional en el Reino Unido y en Estados Unidos que incluyó a 2,035,395 personas de la población general y 99,795 trabajadores de la salud, se identificó que los trabajadores de la salud tienen mayor riesgo que la población general (cociente de riesgo de 11 a 61), siendo más frecuente en sitios donde el uso de equipo personal de protección era inadecuado o inexistente. ${ }^{42}$ Estos datos concuerdan con otros que han indicado que entre 10 y $20 \%$ de las infecciones por COVID-19 ocurren en personal de salud. ${ }^{43,44}$ Hasta septiembre de 2020, cerca de 100,000 trabajadores de la salud en México habían contraído COVID-19, de los cuales 1,320 médicos habían fallecido -la cifra más alta de muertes de personal sanitario a escala internacional-, ${ }^{45,46}$ el uso adecuado de equipo personal de protección parece tener un papel importante, ya que en México como en otros países los trabajadores de limpieza de los centros médicos y hospitales han sido más vulnerables que el personal que está en contacto con pacientes en terapia intensiva. ${ }^{45,47}$

Clínicas y hospitales tendrán que ajustar su operación y procedimientos para enfrentar la pandemia. Algunos espacios no clínicos serán acondicionados para atender enfermos, y personal en retiro o inexperto será reclutado para cubrir vacantes en el proceso de atención. Estas acciones generarán a su vez mayor estrés en el personal de salud, ya que en estos momentos la experiencia del personal será de pri- mordial importancia para disminuir el tiempo que se pase en áreas donde existe riesgo de resultar infectado, de tal forma se ha sugerido, por ejemplo, asignar un equipo de cirujanos con experiencia como los encargados de realizar traqueotomías en pacientes en ventilación mecánica. ${ }^{48}$

Es importante considerar que mientras las autoridades sanitarias en diferentes países establecen políticas para que la población, sobre todo que los mayores de 60 años permanezcan en casa, existe un número importante de personal médico y de enfermería que de no ser parte de la fuerza de trabajadores de la salud permanecerían en casa para disminuir el riesgo de exposición. Si estos médicos y enfermeras se contagian y requieren convalecer en casa u hospitalizados, las consecuencias serían importantes, no sólo por dejar de contar con su experiencia clínica y su presencia cuando son más necesarios, sino por la pérdida de liderazgo, juicio y mantenimiento de la moral del equipo. ${ }^{49}$

Por lo tanto, la ética durante una pandemia requiere de un balance entre servicio y protección personal. El personal de salud enfrentará un riesgo desproporcionado de exposición al COVID-19 en comparación con la población general, muchos temerán adquirir la enfermedad o transmitirla a sus familias, quizá algunos consideren que no deben enfrentar el riesgo y evitarán presentarse a trabajar. ${ }^{3,50}$

La administración de los hospitales y de los sistemas de salud, incluyendo las autoridades sanitarias, deberán proporcionar a su personal de primera línea los equipos e insumos necesarios y respetar su capacidad de decisión sobre la posibilidad de resolver la cascada de enfermos que se presentan solicitando atención, con consideraciones especiales para el cuidado del personal de mayor edad o con condiciones médicas preexistentes, ya que sobre todo los médicos retirados tendrán un papel importante en la medida que avance la pandemia, tal como ha sucedido en Nueva York, el estado de Illinois y la Gran Bretaña. ${ }^{51}$

En México, la protección a las personas en las áreas de trabajo se describen en la norma oficial mexicana NOM-056-SSA1-1993, que establece los requisitos sanitarios del equipo de protección personal en diversas actividades ${ }^{51}$ y la NOM-197-SSA1-2000 que marca los requisitos mínimos de infraestructura y equipamiento de hospitales y consultorios de atención médica especializada; ${ }^{52}$ sin embargo, los artículos 468 y 469 de la Ley General de Salud vigente prevén la aplicación de penas económicas y de privación de la libertad para los trabajadores de la salud que sin causa legítima se rehúsen a desempeñar las fun- 
ciones o servicios que solicite la autoridad sanitaria en ejercicio de la acción extraordinaria en materia de salubridad general. ${ }^{53}$

Por esta aparente antinomia el diputado Edelmiro Santos Santiago Díaz (Médico Ginecólogo y Obstetra, UANL), de la fracción parlamentaria de MORENA, propone una reforma al artículo 470 de la mencionada Ley General con el objetivo de brindar mayor protección al personal al cuidado de la salud durante situaciones de alto riesgo. Esa propuesta legislativa establece incluir en la ley como causa que excluye la responsabilidad del personal sanitario que se niega a actuar -prevista en los artículos 468 y 469 antes mencionados- la ausencia de insumos o condiciones mínimas necesarias para el desarrollo de su actividad, estableciendo en la ley como legítima esa negativa. ${ }^{54}$

Ante esta situación existe un lineamiento ético en el Código para Enfermeras de la Asociación Americana de Enfermeras, de acuerdo con la provisión número 5 de este código, el personal de enfermería tiene la obligación de atender al enfermo si: «1: la ausencia del personal de enfermería expone al paciente a un riesgo significativo de daño, pérdida o lesión; 2: la intervención del personal de enfermería es directamente relevante para prevenir daño al paciente; 3 : los cuidados de enfermería probablemente prevengan daño, pérdida o lesión al paciente; y 4: el beneficio recibido por el paciente es mayor que cualquier riesgo de daño para el personal». ${ }^{5}$ La evaluación de este riesgo debe fundamentarse en evidencia científica vigente relacionada con el uso de equipo de protección y procesos de manejo de infecciones adecuados. ${ }^{3}$

\section{TERAPIA EXPERIMENTAL}

La pandemia del COVID-19 ha puesto una enorme presión en los sistemas de salud. Además de las estrategias de salud pública que tienden a reducir la velocidad y extensión de la propagación de la enfermedad, los médicos deberán tener discusiones de alta calidad para definir las metas de cuidado con los pacientes o sus familias cuando los primeros requieren ser hospitalizados. ${ }^{56}$

La Administración de Alimentos y Fármacos en Estados Unidos (FDA, por sus siglas en inglés) evalúa datos derivados de estudios clínicos con el fin de determinar si los beneficios de un nuevo fármaco superan los riesgos de usarlo. Esta evaluación ha regulado la investigación farmacológica desde 1962 estableciendo estándares de calidad altamente reconocidos ${ }^{57}$ sin embargo, algunos enfermos morirán sin recibir el beneficio de nuevos tratamientos porque el proceso de aprobación puede ser largo y costoso. Por lo anterior y para evitar la muerte de pacientes que sufren de alguna enfermedad, para la cual no existe un tratamiento establecido, la FDA desarrolló un sistema que expande el acceso a tratamientos aun antes de que sean formalmente aprobados. ${ }^{58}$

Con la intensificación de la pandemia nos enfrentamos más a la triste realidad de que no existe un tratamiento específico comprobado más allá de terapia de apoyo. En China, Italia, Francia, España y Estados Unidos un gran número de pacientes ha recibido medicamentos fuera de indicaciones autorizadas o de uso compasivo tales como cloroquina, hidroxicloroquina, azitromicina, lopinavir-ritonavir, favipiravir, ribavirina, interferón, plasma de pacientes convalecientes, esteroides, e inhibidores de la interleucina 6 , con base en su efecto antiviral in vitro o propiedades antiinflamatorias. Fuera de unos cuantos estudios aleatorizados, estas terapias se han utilizado habitualmente sin un grupo control y aun cuando muchos fármacos han demostrado tener actividad in vitro contra diferentes coronavirus, no existe evidencia clínica actual que demuestre la eficacia y seguridad de ninguno de estos fármacos contra ninguna infección por coronavirus en humanos, incluyendo SARS-CoV-2. ${ }^{59}$ Además, independientemente del uso frecuente de antibióticos de amplio espectro para infecciones respiratorias asociadas a COVID-19, existe muy poca evidencia que demuestra la asociación de infecciones nicóticas o bacterianas en estos pacientes. ${ }^{60}$ En un estudio de 836 pacientes hospitalizados por COVID-19 en dos centros nacionales de salud en el Reino Unido se identificaron infecciones bacterianas en $3.2 \%$ de los pacientes en los primeros cinco días y en $6.1 \%$ durante todo el periodo que estuvieron hospitalizados, sin evidencia de infecciones micóticas asociadas al menos en el periodo inicial de la pandemia. ${ }^{61}$ De igual forma, una revisión sistemática de 1,007 abstracts y 18 artículos que analizaron el uso profiláctico de antibióticos de amplio espectro en pacientes con complicaciones respiratorias por COVID-19 publicados hasta abril de 2020 determinó que no había suficiente evidencia de coinfecciones bacterianas o micóticas en estos pacientes. ${ }^{60} \mathrm{~A}$ pesar de ello, una encuesta a 166 médicos en 82 hospitales en 23 países demostró que la mayoría de los encuestados habían utilizado antibióticos en estos pacientes. ${ }^{64}$ Con el fin de evitar consecuencias negativas por el abuso de antibióticos se recomienda enfatizar los principios de administración de antimicrobianos, sobre todo para pacientes con inmunosupresión o con infecciones nosocomiales agregadas. ${ }^{63}$ 
An Med (Mex) 2020; 65 (4): 288-300

A partir del 27 de marzo de 2020, el Departamento de Salud y Servicios Humanos de Estados Unidos declaró la existencia de condiciones que justifican el uso de tratamientos no comprobados para tratar el COVID-19. Inicialmente la FDA autorizó tres medicamentos: fosfato de cloroquina, sulfato de hidroxicloroquina y remdesivir. ${ }^{64}$

El uso de terapias no comprobadas como «último recurso» asume de manera equivocada que el beneficio será mayor que el riesgo. Cuando se administra un medicamento de efecto clínico desconocido a un paciente con una forma severa de una enfermedad nueva no hay forma de saber si el paciente se benefició o sufrió mayor daño sin compararlo con un grupo control concomitante. El uso compasivo de fármacos que no han sido previamente aprobados para uso clínico puede además causar efectos adversos serios que no habían sido detectados con anterioridad debido al uso limitado en pocos pacientes. Por otra parte, una interpretación incorrecta, desafortunadamente frecuente, del uso de medicamentos fuera de indicación o de uso compasivo es atribuir los fallecimientos a la enfermedad y las supervivencias al tratamiento. ${ }^{65,66}$

Además del riesgo de causar daño al paciente sin la posibilidad de detectar siquiera la magnitud de dicho daño, el uso de terapias de uso compasivo o fuera de indicación autorizada y el uso de estudios no controlados durante una pandemia puede desalentar la participación de pacientes y médicos en estudios clínicos aleatorizados afectando negativamente cualquier conocimiento que pudiera obtenerse sobre los efectos de los fármacos estudiados. ${ }^{65}$

Estamos ante una oportunidad inigualable para enfrentar al COVID-19, los hospitales, los médicos, el personal de salud y los líderes profesionales debemos abogar por un abordaje formal para encontrar terapias que pudieran ser efectivas.

\section{BIBLIOGRAFÍA}

1. Lor A, Thomas JC, Barrett DH, Ortmann LW, HerreraGuibert DJ. Key ethical issues discussed at CDC-sponsored international, regional meetings to explore cultural perspectives and contexts on pandemic influenza preparedness and response. Int J Health Policy Manag. 2016; 5 (11): 653662. doi: 10.15171/ijhpm.2016.55.

2. McLean M. Ethical preparedness for pandemic influenza: a toolkit. Markkula Center for Applied Ethics. Oct 1, 2012. 12AD. Available in: www.scu.edu/ethics/focus-areas/bioethics/ resources/ethical-preparedness-for-pandemic-influenza/

3. Payne K. Ethical issues related to pandemic flu planning and response. AACN Adv Crit Care. 2007; 18 (4): 356-360.

4. Strosberg MA. Allocating scarce resources in a pandemic: ethical and public policy dimensions. Virtual Mentor. 2006; 8 (4): 241-244.
5. Walensky RP, Del Rio C. From mitigation to containment of the COVID-19 pandemic: putting the SARS-CoV-2 genie back in the bottle. JAMA. 2020; 323 (19): 1889-1890. doi: 10.1001/ jama.2020.6572.

6. Ferguson NM, Laydon D, Nedjati-Gilani G, Imai N, Ainslie K, Baguelin $\mathrm{M}$ et al. Impact of non-pharmaceutical interventions (NPIs) to reduce COVID-19 mortality and healthcare demand. London: Imperial College COVID-19 Response Team; 2020. doi: https://doi.org/10.25561/77482, accessed 03/17/2020.

7. European Centre for Disease Prevention and Control. COVID-19 situation update worldwide, as of 27 November 2020. [Accessed 9/26/2020] Available in: https://www.ecdc. europa.eu/en/geographical-distribution-2019-ncov-cases

8. Centers for Disease Control and Prevention. 2020. [Accessed 04/11/2020] Available in: https://www.cdc. gov/coronavirus/2019-ncov/covid-data/covidview/pastreports/04172020.html

9. Viglione G. How many people has the coronavirus killed? Nature [Internet]. 2020[accessed 09/26/2020]; 585 (7823): 22-24. doi: 10.1038/d41586-020-02497-w. Available in: https:// www.nature.com/articles/d41586-020-02497-w

10. Roos R. HHS releases new pandemic flu plan. Center for Infectious Disease Research and Policy. 2005. [Accessed 03/08/2020] Available in: https://www.cidrap.umn.edu/newsperspective/2005/11/hhs-releases-new-pandemic-flu-plan

11. Li HO, Bailey A, Huynh D, Chan J. YouTube as a source of information on COVID-19: a pandemic of misinformation? BMJ Glob Health. 2020; 5 (5): e002604. doi: 10.1136/ bmjgh-2020-002604.

12. Pierce J, Randels G. Contemporary bioethics, a reader with cases. New York, Oxford: Oxford University Press; 2010.

13. Department of Veterans Affairs. Meeting the ethical challenges of a severe pandemic influenza. 2010. [Accessed 03/12/2020] Available in: https://www.ethics.va.gov/docs/pandemicflu/ Ethics_and_Pandemic_Flu_Fact_Sheet_508_2010-09-30.pdf

14. Denecke K, Bamidis P, Bond C, Gabarron E, Househ M, Lau $\mathrm{AY}$ et al. Ethical issues of social media usage in healthcare. Yearb Med Inform. 2015; 10 (1): 137-147. doi: 10.15265/IY2015-001.

15. Kuy SR, Gupta R, Correa R, Tsai R, Vohra S. Best practices for a Covid-19 preparedness plan for health systems. NEJM Catal Innov Care Deliv. 2020. doi: 10.1056/CAT.20.0108.

16. APD. ¿Qué es el entorno VUCA y cómo afecta a la supervivencia de las empresas? 2018. [Accessed 04/3/2020] Disponible en: https://www.apd.es/que-es-el-entorno-vuca-ycomo-afecta-a-la-supervivencia-de-las-empresas/

17. Korn Ferry. [accessed 04/27, 2020] Disponible en: https:// www.kornferry.com/insights/articles/burnison-coronavirusleadership-crisis

18. Quinn P. Crisis communication in public health emergencies: the limits of 'legal control' and the risks for harmful outcomes in a digital age. Life Sci Soc Policy. 2018; 14 (1): 4. doi: 10.1186/s40504-018-0067-0.

19. Kim Y. Toward an ethical model of effective crisis communication. Bus Soc Rev. 2015; 120 (1): 57-81.

20. Giuliani RW. Leadership. New York: Miramax books; 2002.

21. Gostin LO, Wiley LF. Governmental public health powers during the COVID-19 pandemic: stay-at-home orders, business closures, and travel restrictions. JAMA. 2020; 323 (21): 2137-2138. doi: 10.1001/jama.2020.5460.

22. Zarzaur BL, Stahl CC, Greenberg JA, Savage SA, Minter $\mathrm{RM}$. Blueprint for restructuring a department of surgery in concert with the health care system during a pandemic: the University of Wisconsin Experience. JAMA Surg. 2020; 155 (7): 628-635. doi: 10.1001/jamasurg.2020.1386. 
23. Aacharya RP, Gastmans C, Denier Y. Emergency department triage: an ethical analysis. BMC Emerg Med. 2011; 11: 16. doi: 10.1186/1471-227X-11-16.

24. Repine TB, Lisagor P, Cohen DJ. The dynamics and ethics of triage: rationing care in hard times. Mil Med. 2005; 170 (6): 505-509

25. Iserson KV, Moskop JC. Triage in medicine, part I: Concept, history, and types. Ann Emerg Med. 2007; 49 (3): 275-281.

26. Beauchamp TL, Childress JF. Principles of biomedical ethics. New York: Oxford University Press; 2009.

27. Bernstein SL, Aronsky D, Duseja R, Epstein S, Handel D, Hwang $U$ et al. The effect of emergency department crowding on clinically oriented outcomes. Acad Emerg Med. 2009; 16 (1): 1-10.

28. Hodge AN, Marshall AP. Violence and aggression in the emergency department: a critical care perspective. Aust Crit Care. 2007; 20 (2): 61-67.

29. Ekwall A, Gerdtz M, Manias E. The influence of patient acuity on satisfaction with emergency care: perspectives of family, friends and carers. J Clin Nurs. 2008; 17 (6): 800-809.

30. Hartman RG. Tripartite triage concerns: issues for law and ethics. Crit Care Med. 2003; 31 (5 Suppl): S358-S361. doi: 10.1097/01.CCM.0000065130.18337.05.

31. White DB, Lo B. A framework for rationing ventilators and critical care beds during the COVID-19 pandemic. JAMA. 2020; 323 (18): 1773-1774. doi: 10.1001/jama.2020.5046.

32. Curtis JR, Kross EK, Stapleton RD. The importance of addressing advance care planning and decisions about do-notresuscitate orders during novel coronavirus 2019 (COVID-19). JAMA. 2020; 323 (18): 1771-1772. doi: 10.1001/jama.2020.4894.

33. https://www.health.pa.gov/topics/Documents/Diseases $\% 20$ and $\% 20$ Conditions/COVID-19\%20Interim $\% 20$ Crisis $\% 20$ Standards\%20of\%20Care.pdf (accessed 12/09/2020)

34. Miceli M. Bioethics in practice: unilateral do-not-resuscitate orders. Ochsner J. 2016; 16 (2): 111-112.

35. Wallace CL, Wladkowski SP, Gibson A, White P. Grief during the COVID-19 pandemic: considerations for palliative care providers. J Pain Symptom Manage. 2020; 60 (1): e70-e76.

36. Janssen DJA, Ekström M, Currow DC, Johnson MJ, Maddocks M, Simonds AK et al. COVID-19: guidance on palliative care from a European Respiratory Society international task force. Eur Respir J. 2020; 56 (3): 2002583.

37. Fusi-Schmidhauser T, Preston NJ, Keller N, Gamondi C. Conservative management of COVID-19 patients-emergency palliative care in action. J Pain Symptom Manage. 2020; 60 (1): e27-e30

38. Chidiac C, Feuer D, Naismith J, Flatley M, Preston N. Emergency palliative care planning and support in a COVID-19 pandemic. J Palliat Med. 2020; 23 (6): 752-753.

39. Kaiser Health News. Palliative care helped family face 'the awful, awful truth'. 2020. [Accessed 05/11/2020] Available in: https://khn.org/news/palliative-care-helped-family-face-theawful-awful-truth/

40. Centers for Disease Control and Prevention. Cost of the Ebola epidemic. 2019. [Accessed 03/30/2020] Available in: https:// www.cdc.gov/vhf/ebola/history/2014-2016-outbreak/cost-ofebola.html

41. Beckman AL, Gondi S, Forman HP. How to stand behind frontline health care workers fighting coronavirus. Health Affairs; 2020. [Accessed 03/30/2020] Available in: https://www. healthaffairs.org/do/10.1377/hblog20200316.393860/full/

42. Nguyen LH, Drew DA, Graham MS, Joshi AD, Guo CG, Ma W et al. Risk of COVID-19 among front-line health-care workers and the general community: a prospective cohort study. Lancet Public Health. 2020; 5 (9): e475-e483.
43. Lazzerini M, Putoto G. COVID-19 in Italy: momentous decisions and many uncertainties. Lancet Glob Health. 2020; 8 (5): e641-e642.

44. CDC COVID-19 Response Team. Characteristics of health care personnel with COVID-19-United States, February 12-April 9, 2020. MMWR Morb Mortal Wkly Rep. 2020; 69 (15): 477-481.

45. México es el país que registra más muertes de trabajadores de salud por Covid-19. El Economista [Internet]. 03 de septiembre de 2020. [Acceso 09/26/2020] Disponible en: https://www.eleconomista.com.mx/politica/Mexico-es-el-paisque-registra-mas-muertes-de-trabajadores-de-salud-porCovid-19--20200903-0037.html

46. Agren D. Understanding Mexican health worker COVID-19 deaths. Lancet. 2020; 396 (10254): 807.

47. Shields A, Faustini SE, Perez-Toledo M, Jossi S, Aldera E, Allen JD et al. SARS-CoV-2 seroprevalence and asymptomatic viral carriage in healthcare workers: a cross-sectional study. Thorax. 2020; 75 (12): 1089-1094.

48. Tay JK, Khoo ML, Loh WS. Surgical considerations for tracheostomy during the COVID-19 pandemic: lessons learned from the severe acute respiratory syndrome outbreak. JAMA Otolaryngol Head Neck Surg. 2020; 146 (6): 517-518.

49. Buerhaus PI, Auerbach DI, Staiger DO. Older clinicians and the surge in novel coronavirus disease 2019 (COVID-19). JAMA. 2020; 323 (18): 1777-1778. doi: 10.1001/jama.2020.4978.

50. Livingston E, Desai A, Berkwits M. Sourcing personal protective equipment during the COVID-19 pandemic. JAMA. 2020; 323 (19): 1912-1914. doi: 10.1001/jama.2020.5317.

51. NORMA Oficial Mexicana NOM-056-SSA1-1993, Requisitos sanitarios del equipo de protección personal. [Acceso 03/12/2020] Disponible en: http://www.salud.gob.mx/unidades/ cdi/nom/056ssa13.html

52. NORMA Oficial Mexicana NOM-197-SSA1-2000, Que establece los requisitos mínimos de infraestructura y equipamiento de hospitales y consultorios de atención médica especializada. [Acceso 03/12/2020] Disponible en: http://www. salud.gob.mx/unidades/cdi/nom/197ssa10.html

53. JUSTIA México. [Acceso 03/15/2020] Disponible en: https:// mexico.justia.com/federales/leyes/ley-general-de-salud/titulodecimo-octavo/capitulo-vi/\#articulo-468

54. Santiago Díaz ES. Proyecto de Decreto por el que se reforma el artículo 470 de la Ley General de Salud. Gaceta Parlamentaria, año XXIII; número 5502-1; 21 de abril de 2020; páginas 43-49. Cámara de Diputados LXIV Legislatura. Disponible en: https:// bit.ly/2Wx96c1 (HTML) y https://bit.ly/3cML3vD (PDF).

55. American Nurses Association. Code of ethics for nurses with interpretive statements. Washington, DC: American Nurses Association; 2001.

56. Meagher KM, Cummins NW, Bharucha AE, Badley AD, Chlan LL, Wright RS. COVID-19 ethics and research. Mayo Clin Proc. 2020; 95 (6): 1119-1123. doi: 10.1016/j. mayocp.2020.04.019.

57. Darrow JJ, Sarpatwari A, Avorn J, Kesselheim AS. Practical, legal, and ethical issues in expanded access to investigational drugs. N Engl J Med. 2015; 372 (3): 279-286. doi: 10.1056/ NEJMhle1409465.

58. Okie S. Access before approval--a right to take experimental drugs? N Engl J Med. 2006; 355 (5): 437-440. doi: 10.1056/ NEJMp068132.

59. Goodman JL, Borio L. Finding effective treatments for COVID-19: scientific integrity and public confidence in a time of crisis. JAMA. 2020; 323 (19): 1899-1900. doi: 10.1001/ jama.2020.6434. 
An Med (Mex) 2020; 65 (4): 288-300

60. Rawson TM, Moore LSP, Zhu N, Ranganathan N, Skolimowska $\mathrm{K}$, Gilchrist $\mathrm{M}$ et al. Bacterial and fungal co-infection in individuals with coronavirus: A rapid review to support COVID-19 antimicrobial prescribing. Clin Infect Dis. 2020: ciaa530. doi: 10.1093/cid/ciaa530.

61. Hughes S, Troise O, Donaldson H, Mughal N, Moore LSP. Bacterial and fungal coinfection among hospitalized patients with COVID-19: a retrospective cohort study in a UK secondary-care setting. Clin Microbiol Infect. 2020; 26 (10): 1395-1399.

62. Beović B, Doušak M, Ferreira-Coimbra J, Nadrah K, Rubulotta F, Belliato M et al. Antibiotic use in patients with COVID-19: a 'snapshot' infectious diseases international research initiative (ID-IRI) survey. J Antimicrob Chemother. 2020; 75 (11): 3386-3390.
63. Clinical management of severe acute respiratory infection (SARI) when COVID-19 disease is suspected interim guidance. World Health Organization website. Published May 27, 2020. [Accessed 09/26/2020] Available in: http:// apps.who.int/iris/bitstream/10665/178529/1/WHO_MERS_ Clinical_15.1_eng.pdf.

64. Ison $\mathrm{MG}$, Wolfe C, Boucher HW. Emergency use authorization of remdesivir: the need for a transparent distribution process. JAMA. 2020; 323 (23): 2365-2366. doi: 10.1001/jama.2020.8863.

65. Loutfy M. Changing the rules in times of crisis: do desperate times allow desperate measures? Virtual Mentor. 2006; 8 (4): 214-218.

66. Kalil AC. Treating COVID-19-off-label drug use, compassionate use, and randomized clinical trials during pandemics. JAMA. 2020; 323 (19): 1897-1898. doi: 10.1001/jama.2020.4742. 